\title{
Gradient porosity poly(dicyclopentadiene)
}

\author{
A. Della Martina and J.G. Hilborn ${ }^{\text {a),b) }}$ \\ Polymers Laboratory, Materials Department, Swiss Federal Institute of Technology, \\ CH-1015 Lausanne, Switzerland
}

(Received 27 December 2000; accepted 2 May 2001)

This article describes the preparation of gradient porosity thermoset polymers. The technique used is based on polymerizing a solution of cross-linkable dicyclopentadiene and 2-propanol. The forming polymer being insoluble in 2-propanol, phase separation occurs. Subsequent drying of the 2-propanol gives porosities up to $80 \%$. An apparatus was built to produce a gradient in 2-propanol concentration in a flask, resulting in polymerized gradient porosity rods. The resulting materials have been characterized by scanning electron microscopy (SEM) and density measurements. A mathematical model which allows prediction of the gradient produced is also presented.

\section{INTRODUCTION}

Functional graded materials are materials engineered with gradual transitions in microstructure and/or composition. The motivation for producing gradient materials comes from functional performance requirements varying with position within the element. ${ }^{1,2}$ Graded metals and metal-ceramic composites have been widely studied for extremely demanding structural applications, such as aerospace or internal parts of high performance engines. ${ }^{3}$ Indeed such materials were mainly designed for increased resistance to thermal stresses. The different methods known to produce gradient materials can be classified in two categories: constructive processes and transport based processes. ${ }^{2}$ The first type consists in building the gradient, layer by layer, with the appropriate distribution of constituents. The second relies on natural transport phenomena such as diffusion or heat transfer to create the gradient. Gradient porosity materials have been produced mainly using spray coating ${ }^{4}$ or powder technology. ${ }^{5-7}$ Other reported techniques include selfpropagating high-temperature synthesis ${ }^{8}$ or electrochemical modification of porous preforms. ${ }^{9}$

Functionally gradient composites containing polymers have also been investigated. For example an epoxy/ carbon fiber gradient composite has been produced using a centrifugal method, ${ }^{10}$ and polyimide/aluminum and polyimide/copper gradient composites have been made by powder technology. ${ }^{11,12}$ For polymer/polymer systems the most important application is gradient refractive index optical fibers. ${ }^{13}$ However, very little work has been

\footnotetext{
a) Address all correspondence to this author.

b) Present address: The Ångstrom Laboratory, Uppsala University, S-75121 Uppsala, Sweden.
}

published in this area, and attention must be paid to terminology. The expression gradient polymers designates either a gradient in concentration of one monomer along a single chain of copolymer (also more adequately termed gradient copolymers),${ }^{14-16}$ or functionally graded polymeric materials (FGPM). The main techniques for producing FGPM are diffusion polymerization ${ }^{14-17}$ and powder technology combined with compression molding. ${ }^{18}$ The first is limited to thin parts, and the second is difficult to apply to polymers and limited, for compaction reasons, to symmetrical shapes. Graded porosities are nevertheless quite common in polymers, density gradients often being induced, more or less intentionally, by foaming processes such as reaction injection molding ${ }^{19}$ or phase inversion techniques. ${ }^{20}$ The porosity gradients obtained with these two techniques arise from transportbased processes. To our knowledge no engineered gradient porosity polymers, produced via a constructive process, have been reported.

Recently we produced macroporous poly(dicyclopentadiene) (PDCPD) via chemically induced phase separation (CIPS). ${ }^{21}$ This technique consists of mixing a polymerizable monomer with a nonreacting liquid substance, which can be a low molecular weight liquid, a polymer, etc. The enthalpic and entropic changes of the system due to the polymerization of the monomer induce a change in solubility. If this substance is carefully chosen, the solubility change can be large enough for this substance to be soluble with the monomer but nonsoluble with the polymer. Such a substance will thus separate from a resulting polymer-rich phase generating a twophase morphology. This principle has been described earlier as chemical cooling, ${ }^{22}$ polymerization-induced phase separation, ${ }^{23}$ reaction-induced phase separation. ${ }^{24-27}$ Epoxy and cyanate systems with well-defined porosity have been produced after extraction of the 
separated substance (low molecular weight liquids can be extracted by drying for example). ${ }^{28-31}$ This substance is, in such cases, often referred to as a porogen, as it will template the final porosity.

In the case of highly cross-linked polymers such as divinylbenzene and PDCPD, the enthalpic and entropic changes induced by polymerization are large enough to induce phase separation over a wide range of initial porogen concentrations. This allows the preparation of materials with closed porosity for low initial porogen concentrations and up to $80 \%$ open porosity for high porogen concentrations. ${ }^{21}$ Thus, by creation of a gradient in porogen concentration in the initial mixture, a full range of porosities can be envisaged in a single sample.

To create this gradient, a technique similar to that used to produce gradient density columns has been adapted. The aim of the present work is to study the feasibility of this novel approach. The resulting gradient porosity bars are also anticipated to be helpful in understanding the phase separation mechanisms occurring with CIPS. Indeed, the concentration gradient produced along the sample allows monitoring of all the possible phase separation behaviors for the chosen polymerization conditions. The resulting materials will be characterized by density measurements and scanning electron microscopy (SEM).

\section{EXPERIMENTAL}

\section{A. Materials}

Dicyclopentadiene (DCPD: "Petroplast 94\%" from Shell, 94\% pure, mainly endo and containing stabilizer, trimers, and other products) was degassed (5 min under 1 mbar pressure and then purged with $\mathrm{N}_{2}$ ) and stored over 5-Å molecular sieves. 2-Propanol, dichloromethane, and $1,1^{\prime}, 2,2^{\prime}$-tetrachloroethane (purum) were used as received. The catalyst, $\mathrm{RuCl}_{2}\left(\mathrm{p}-\mathrm{MeC}_{6} \mathrm{H}_{4} \mathrm{CHMe}_{2}\right)\left(\mathrm{PCy}_{3}\right)$, where $\mathrm{Cy}=$ cyclohexyl and abbreviated Ru-cat, was synthesized, according to Bennett and $\mathrm{Smith}^{32}$ and kindly supplied by A. Mühlebach (Ciba Specialty Chemicals).

\section{B. Preparation of the samples}

On the basis of the general procedures for building liquid-liquid gradients, ${ }^{33}$ the samples were produced as follows:

First the setup shown in Fig. 1 was prepared. This setup consisted of two stacked $30 \mathrm{ml}$ Luer Syringes, the top one directly pouring into the bottom one. A $90^{\circ}$-tilted magnetic stirrer was mounted against the bottom syringe to induce the spinning of the magnetic stir bar immersed in it. The bottom syringe poured in a disposable $45 \mathrm{ml}$ screw cap flask. Initially, the syringe needles were plugged.

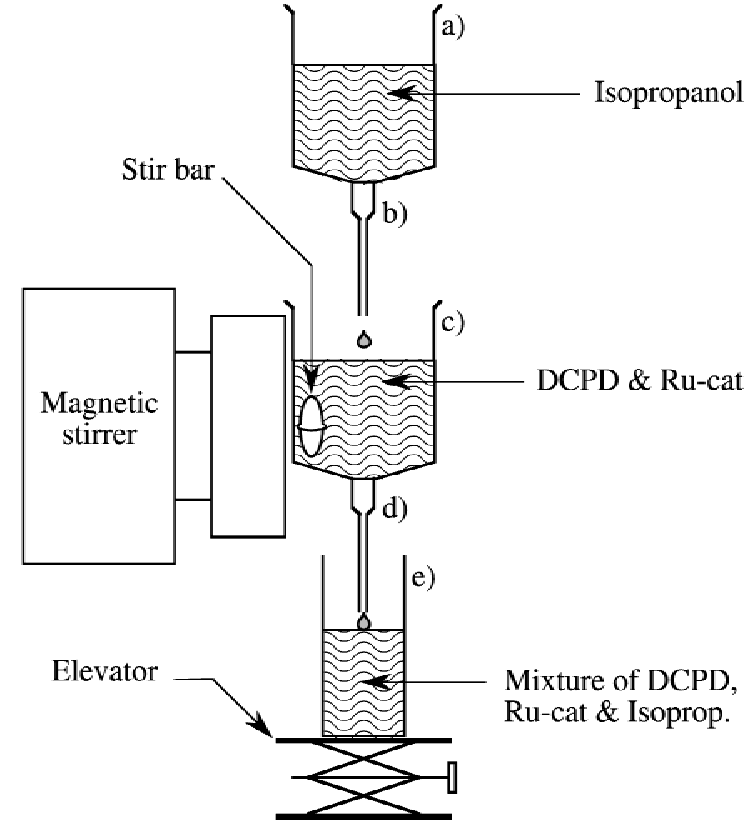

FIG. 1. Schematic representation of the experimental setup used to produce gradient porosity poly(dicyclopentadiene) bars: (a) top syringe; (b) top needle; (c) bottom syringe; (d) bottom needle; (e) flask.

Solutions of $10 \mathrm{wt} \% \mathrm{Ru}$-cat in either dichloromethane (for samples 1, 3, and 4) or 1,1',2,2'-tetrachloroethane (for sample 2) were prepared. A 20-ml volume of DCPD was then added to $2 \mathrm{ml}$ of catalyst solution. For samples $\mathbf{3}$ and $\mathbf{4}$ only, after solubilization of the Ru-cat in the DCPD, the dichloromethane was evaporated by placing the mixture under vacuum until no more boiling was observed. The mixtures were added into the bottom syringe, and $20 \mathrm{ml}$ of 2-propanol was added into the top syringe. The stirrer was then switched on and the needle stoppers removed. To avoid mixing in the receiving flask, the tip of the bottom needle was kept against the surface of the mixture by lowering the flask with the elevator.

After formation of the gradient, the flasks were sealed and placed for polymerization in a preheated oven at $80{ }^{\circ} \mathrm{C}$ for $20 \mathrm{~h}$. The flasks were then broken and the samples dried under vacuum in an oven at $84{ }^{\circ} \mathrm{C}$, for 3 days.

\section{Characterization techniques}

Samples 1 and 4 were sawed using a disk saw $(0.5 \mathrm{~mm}$ thick) to obtain regular disks of $2-\mathrm{mm}$ thickness. The density of the disks was obtained either by weighing them and determining their volume with a caliper (open porosity disks) or by using a Mettler Density Measurement Kit adapted to an AT261 DeltaRange balance (closed porosity disks). Sample 3 was notched along the axis and fractured at liquid-nitrogen temperature. 
The fracture surfaces were gold sputtered with a BIO-RAD Polar Division SEM Coating System, Sputter Coated E5400, operated at $1 \mathrm{kV}$ with $20 \mathrm{~mA}$ current for $60 \mathrm{~s}$ to obtain a gold layer of $20 \mathrm{~nm}$ approximately. The gold-sputtered fracture surfaces were observed by SEM using a Phillips XLF-30 microscope equipped with a field emission gun operated at $2 \mathrm{kV}$.

\section{RESULTS AND DISCUSSION}

\section{A. Preparation of the samples}

The experimental setup was first tested with pure DCPD (i.e., without catalyst) to match the flows of the top and bottom syringe, to produce the desired concentration gradient in the receiving flask. To empty the two syringes simultaneously, the flow through the bottom needle has to be approximately twice the flow through the top one. Ideally, the bottom syringe should empty just before the top one. Thus, at the end of the mixing, some pure 2-propanol is present at the top of the flask, indicating that the whole range of concentrations is obtained. This was achieved using smaller needles for the top syringe as the 2-propanol is also less viscous than the $\mathrm{DCPD}+\mathrm{Ru}$-cat $+\mathrm{CH}_{2} \mathrm{Cl}_{2}$ mixture. After trying different needle sizes, the best conditions for our setup were obtained with a $40 \times 0.8 \mathrm{~mm}$ top needle and a $40 \times 0.9 \mathrm{~mm}$ bottom needle.

Ruthenium-based catalysts are known to possess remarkable tolerance toward most functional groups, ${ }^{34-36}$ including protic compounds, which would make them suitable candidates when using porogens such as 2-propanol. Previous work ${ }^{37-39}$ has shown that ruthenium arene phosphine complexes \{of type $\left[\mathrm{Ru}(\right.$ arene $\left.) \mathrm{Cl}_{2}\left(\mathrm{PR}_{3}\right)\right]$, where $\mathrm{R}$ can be cyclohexyl, as used in this work, or isopropyl $\}$ are very efficient catalysts for the ringopening metathesis polymerization (ROMP) of technical grade DCPD, providing it contains acetylenic impurities. This catalyst is efficient at temperatures of $80^{\circ} \mathrm{C}$ and higher, and the system possesses an excellent latency at room temperature (gelation takes several hours). This latency allows for the mixing and processing (production of the gradient in the flask) necessary before polymerization. The flasks in which the gradient solution has been prepared must be carefully handled when sealed and during placement in the oven for polymerization. It is also important to close the flasks hermetically to allow polymerization temperatures above the boiling point of 2-propanol, thus avoiding solvent loss or bubble formation inside the samples. For this work, the lowest efficient temperature, $80^{\circ} \mathrm{C}$, giving slow polymerization and allowing ample time for phase separation during polymerization, has been chosen.

The final porous samples were obtained after drying in a vacuum oven at $80{ }^{\circ} \mathrm{C}$ for 3 days. This temperature was chosen to be well below the glass transition of the PDCPD matrix, thus avoiding softening which could result in collapse of the foam structures.

The polymerization along with the accompanying phase separation (CIPS technique) of the gradient concentration DCPD-2-propanol system resulted in gradient porosity bars, after removal of the 2-propanol. These measured approximately $25 \mathrm{~mm}$ in diameter and $60 \mathrm{~mm}$ in length. Other dimensions are feasible using other setups and different amounts of products, but attention must be paid in adjusting the flow conditions to obtain reasonable processing times. Processing times should not be too fast to allow for good mixing. On the other end, processing times are not limited, except for the operator's comfort, as the latency of the catalyst should allow for even hours-long processing. Large gradient pieces, for example, should be feasible with scaled equipment, and particularly larger needles, to avoid too long processing times. Equation (9) of Appendix A gives the initial volumetric concentration of 2-propanol along the sample for a setup similar to the one used in this work (Fig. 1). With the matching of the parameters of this equation to the final desired material, any monotonic gradient can be designed. Moreover, if the desired gradient should start with a fixed 2-propanol concentration $\neq 0$ (i.e. a final bar starting with a desired non-null porosity), Eq. (9) can be recalculated with different initial conditions when solving Eq. (4).

When sample 1 was sawed for density measurements, macroscopic holes were observed inside the bar, resulting from the boiling of dichloromethane during the polymerization of the DCPD. To avoid this, tetrachloroethane, which has a higher boiling point, was used for sample 2. However, sample 2 remained soft after polymerization, owing to plasticization of the matrix by the tetrachloroethane. In this case, the plasticized matrix was not rigid enough to support the surface tensions produced by drying. These induced a collapse of the microstructures formed by phase separation, resulting in macroscopic shrinkage of the bar. Finally, it was decided to use dichloromethane to solubilize the catalyst in the DCPD, and when a homogeneous solution was obtained, the dichloromethane was evaporated before using the mixture to build the gradient. After complete evaporation of the dichloromethane, i.e., when no more boiling was observed under vacuum, no precipitation of the catalyst in the DCPD was observed.

Samples $\mathbf{3}$ and $\mathbf{4}$ were not perfectly regular, the crosssection being slightly different along the axis of the bars. These variations arise from the different phase separation mechanisms and the different final morphologies produced in the sample, depending on the initial local 2-propanol concentration. At the bottom of the bars, i.e., at low 2-propanol concentrations, precipitation of 2-propanol droplets into a PDCPD matrix occurs. In this 
dynamic process which is in competition with the polymerization of the matrix, some 2-propanol is trapped into the matrix and then extracted during the drying step, resulting in shrinkage of the matrix. Higher in the bar, at higher 2-propanol concentrations, PDCPD droplets precipitate into liquid 2-propanol. Since the diffusion of the precipitating substance is not limited, the phase separation is almost complete. In this case, the final PDCPD contains very little 2-propanol and the shrinkage on drying is therefore negligible. As the limit between these different parts of the bars might not be always perfectly perpendicular to the axis (presumably due to some convection inside the flask), the different shrinkage of the different parts of the bars might induce some slight tilting of the bars, as could be seen with sample $\mathbf{1}$.

\section{B. Density of the gradient rod}

One of the goals of this work was to be able to design the final gradient. Therefore, the gradient porosity materials will be compared with an expected density estimated as follows. Using density results obtained earlier with bulk samples of fixed 2-propanol concentration, ${ }^{21}$ a theoretical porosity gradient can be deduced from the calculated 2-propanol concentration gradient Fig. 2.

First the 2-propanol volumetric concentration profile set in the flask can be plotted using Eq. (9) (Appendix A) with the measured top and bottom flow rates, the initial volume in the bottom syringe, and the real dimensions of the flask: $Q_{\mathrm{b}}=2.1168 \mathrm{ml} / \mathrm{min}=35.28 \mu \mathrm{l} / \mathrm{s} ; Q_{\mathrm{t}}=1$ $\mathrm{ml} / \mathrm{min}=16.67 \mu \mathrm{l} / \mathrm{s} ; V_{0}=19.8 \mathrm{ml}=19^{\prime} 800 \mu \mathrm{l}$; $r_{\mathrm{f}}=12.7 \mathrm{~mm}$.

The density obtained earlier with bulk samples is plotted in Fig. 3 as a function of the initial 2-propanol concentration. Two linear functions have been fitted to these results, one for each of the two mechanisms of phase separation, namely the precipitation of 2-propanol

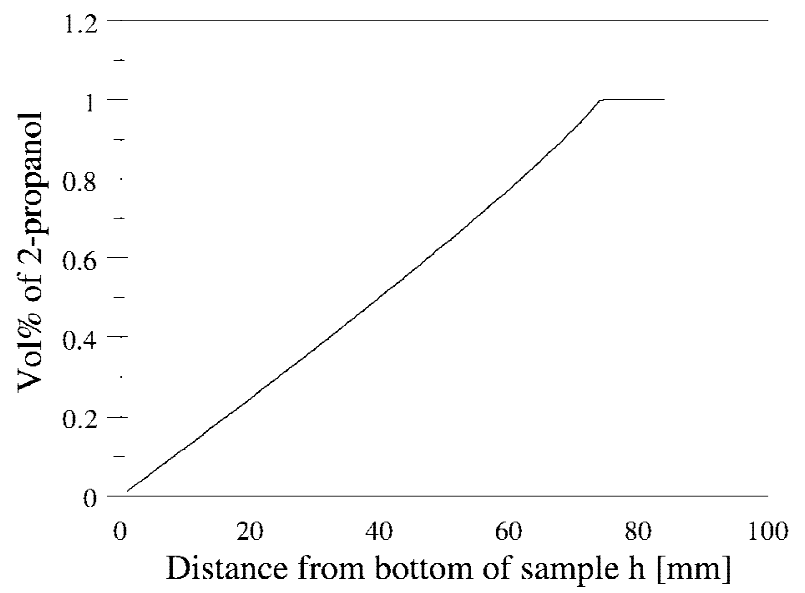

FIG. 2. Predicted volumetric concentration of 2-propanol along the axis of the samples. droplets into a PDCPD matrix and the precipitation and agglomeration of PDCPD particles into the 2-propanol. These two mechanisms have different kinetics, so that the final densities produced by CIPS show different linear dependence on composition.

Applying the fitted linear dependences form in Fig. 3 to the concentration profile given in Fig. 2 gives an estimation of the density that should be found along the samples. This estimated density profile and the density measurements performed on the slices of sample $\mathbf{4}$ are given in Fig. 4.

The functions in Figs. 3 and 4 are shown for the concentration domains for which bulk samples were made, without extrapolation.

The measured density along the sample roughly follows the predicted gradient. The first $10 \mathrm{~mm}$ of the bar, however, does not show measurable decrease in density

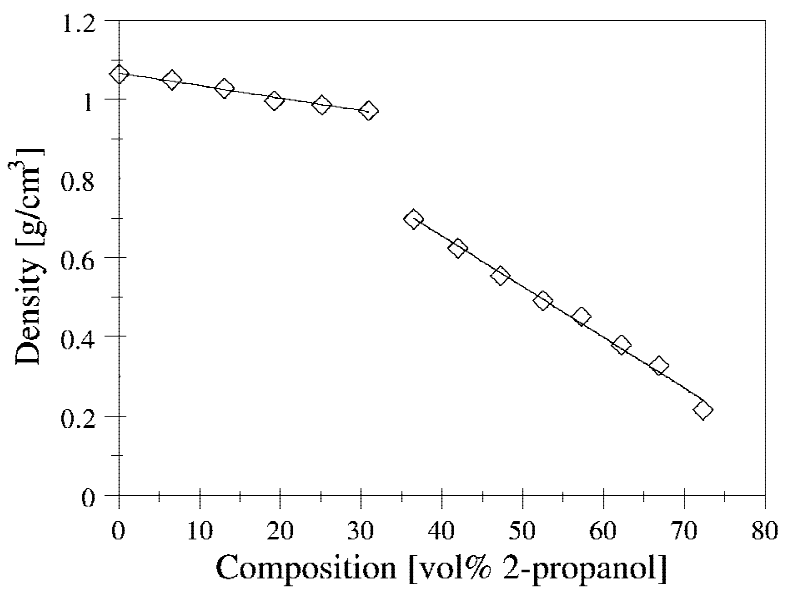

FIG. 3. Densities measured and linear fitting for the bulk samples. The linear functions are the following: for the low 2-propanol contents, density $=1.0654-0.0031558(\mathrm{vol} \% 2$-propanol $), R=0.99249$; for the high 2-propanol contents, density $=1.1653-0.012782(\mathrm{vol} \%$ 2-propanol), $R=0.996$.

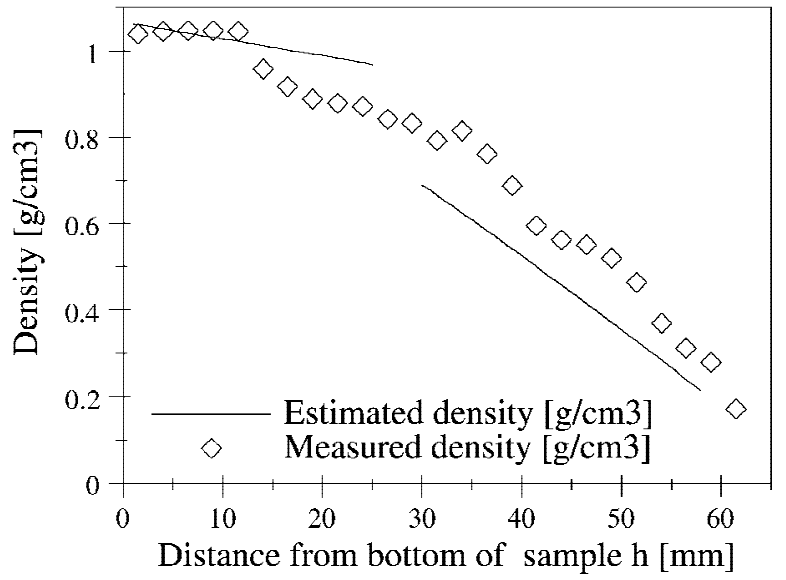

FIG. 4. Estimated density profile along the axis of the samples (continuous line) and density of sample 4 (lozenges). 
(result confirmed by the lack of pores observed by SEM). This suggests that the initial stages of the mixing in the bottom syringe, when a large volume of mixture is processed, are not very efficient. If the 2-propanol that has poured form the beginning of the mixing has not formed porosity in the produced gradient, it must have accumulated in the bottom syringe and the concentration of 2-propanol must have increased in a certain fraction of the volume of the mixture (very likely the top one). When that higher 2-propanol concentration fraction of mixture pours in the flask, a jump in porosity is expected. This density drop is shown by Fig. 4, at $h \approx 13 \mathrm{~mm}$. The measured density then decreases as predicted. For the same reasons, the transition between the two regimes of phase separation (2-propanol droplets precipitation and PDCPD particles precipitation) is also shifted higher in the flasks than expected. According to the densities measured for the slices of sample 4, this transition occurs just above $h=30 \mathrm{~mm}$, an observation confirmed by SEM. Nevertheless, the first bulk sample with an agglomerated particles morphology was obtained for $36.5 \mathrm{vol} \%$ 2-propanol. According to the predicted 2-propanol gradient, this concentration should have been found at around $h=29.5 \mathrm{~mm}$. This implies that this transition should have occurred before $h=29.5 \mathrm{~mm}$. Moreover, this transition was expected to be accompanied by a drop in density, whereas the measured density profile for the bar is continuous. Higher in the bar the measured densities again decrease roughly according to the prediction.

Some of the discrepancy between the predicted density gradient and the measured density profile might also arise from convection, which mixes fractions of solution of different concentration, in the flask during the early stages of the polymerization, when the viscosity is low.
This convection can probably be avoided by adding a nonreacting polymer in the mixture, thus increasing the viscosity of the system. Attention must be paid to the final microstructure, however, as the increase in viscosity might slightly change the phase separation behavior. Of course if the viscosity is increased too much, the setup used in the present work, which takes advantage of gravity for mixing the initial components and filling the receiving flask, might not work. In this case, as well as for industrial scale up, a system with pumps to feed the mixer and to fill the mold can be envisaged. Better mixing conditions should also probably allow better fit of the resulting gradient to the prediction given by the model.

\section{Microstructure of the gradient rod}

Five SEM pictures of the morphology along sample $\mathbf{3}$ are given in Fig. 5. The observed microstructures suggest nucleation and growth phase separation mechanisms. At low 2-propanol concentration, i.e., at short distances from the bottom of the sample, the precipitation of 2-propanol droplets in a continuous PDCPD matrix can be deduced. As the concentration of 2-propanol increases, together with distance from the bottom of the sample, the fraction of porosity increases. The estimated 2-propanol concentration for $h=26 \mathrm{~mm}$ [Fig. 5(c)] corresponds to a concentration for which no result could be deduced from the bulk samples (blank region in the middle of the graph shown in Fig. 4). The critical point, i.e., the point separating the two nucleation and growth regimes (2-propanol droplets into a polymer-rich phase and PDCPD droplets into 2-propanol-rich solution) was expected to be in this region. It can be seen that as the concentration of 2-propanol approaches this critical point, the porosity increases dramatically. Higher in the bar and above the critical point, the reversed precipitation

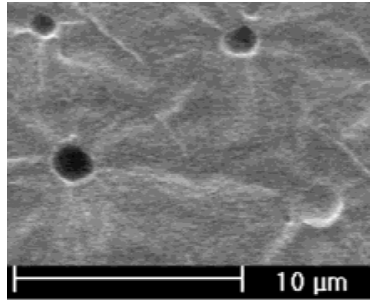

(a)

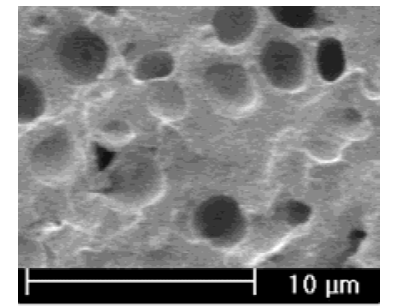

(b)

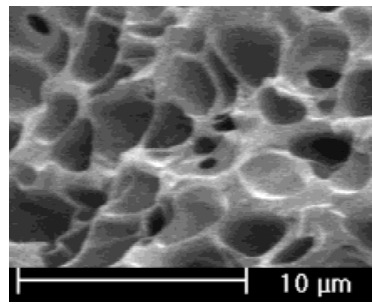

(c)

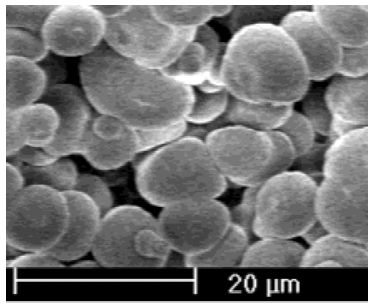

(d)

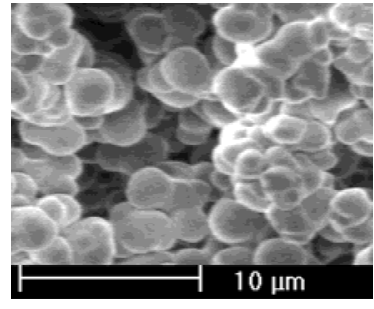

(e)

FIG. 5. Microstructure along the axis of the bar: (a) $h=10 \mathrm{~mm}$; (b) $h=24.5 \mathrm{~mm}$; (c) $h=26 \mathrm{~mm}$; (d) $h=37.4 \mathrm{~mm}$; (e) $h=48.9 \mathrm{~mm}$. 
mechanism apparently occurs. Precipitation of PDCPD droplets in 2-propanol leads to the formation of a morphology consisting of agglomerated particles.

However, the cocontinuous morphology which might result from spinodal decomposition was never observed. For the 2-propanol composition of $30.9 \mathrm{vol} \%$, the bulk sample showed precipitated 2-propanol droplets in PDCPD. Our calculated model predicted that this concentration should have been found at $h=25 \mathrm{~mm}$ in the gradient bar. In the bulk sample with 36.5 vol\% 2-propanol, concentration expected at $h=30 \mathrm{~mm}$ in the bar, agglomeration of PDCPD particles was observed. This suggested that eventual spinodal decomposition should have occurred between these heights in the gradient bar. Figure 6 shows four SEM pictures of that region.

In the region where spinodal decomposition was expected, an interpenetration of the two morphologies resulting from nucleation and growth can be observed. The commonly accepted generic phase diagram of chemically induced phase separation for this system ${ }^{21}$ suggests that, with equilibrium conditions, spinodal decomposition should occur for at least one composition. Spinodal decomposition is a phase separation mechanism that does not proceed via nucleation and growth and, thus, does not have to overcome a nucleation energy to start. Polymerrich and porogen-rich regions are formed by local fluctuations of the concentration. The low polymerization temperature used here gives a slow polymerization rate, and when the spinodal decomposition starts, time enough is given to the system to promote nucleation within these regions in which the porogen concentration is shifted away from the critical composition. Then, as the nucleated droplets/particles have a higher growth rate than the spinodal morphology, they overcome it and finally occupy all the volume. The higher growth rate of the nucleated droplets/particles arises from the diffusion rates of the 2-propanol with respect to PDCPD, which are higher in the case of the formation of the morphology arising from nucleation and growth than for the building of the spinodal morphology. In the first case, diffusion follows the concentration gradient as in the second; the diffusion must go against the concentration gradient. ${ }^{40}$

\section{CONCLUSION}

A novel technique to prepare gradient porosity materials has been described. This technique is based on chemically induced phase separation of a gradient concentration mixture obtained using an own-developed apparatus, based on a technique similar to that used to produce gradient columns. This apparatus consists of two syringes one pouring 2-propanol in the one containing the precursors of the PDCPD, a stir bar being set in the latter, pouring the mixture into a sealable flask. The key requirements to the success of this technique are the choice of the porogen, which has to be soluble with the monomer but insoluble with the polymer, the resistance of the catalyst to the porogen, and the adjustments of the flow conditions to give the desired final gradient. The final porosities produced ranged from 0 up to $83 \%$, with open morphologies for total porosities of $20 \%$ and up. A

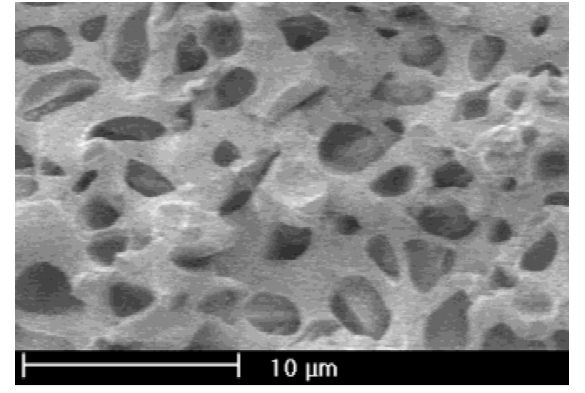

(a)

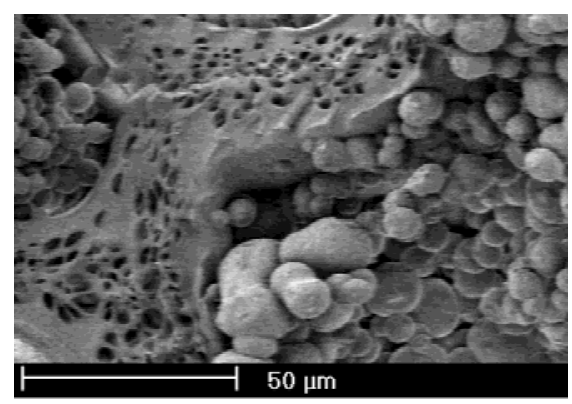

(c)

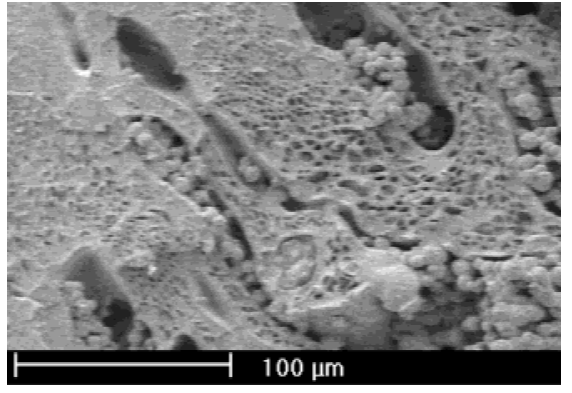

(b)

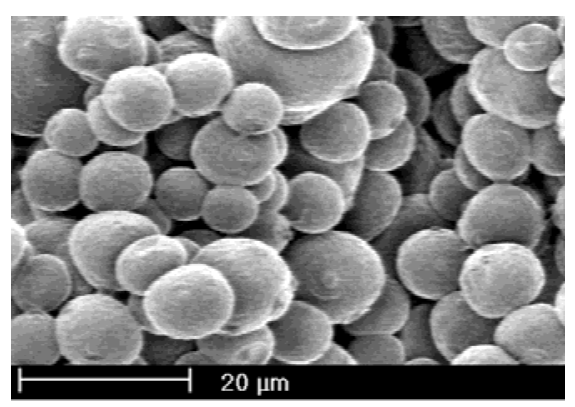

(d)

FIG. 6. Microstructure along the axis of the bar: (a) $h=29.5 \mathrm{~mm}$; (b) $h=30 \mathrm{~mm}$; (c) $h=30.3 \mathrm{~mm}$; (d) $h=30.8 \mathrm{~mm}$. 
model is presented describing the gradient formation in the bar. This model allows an approximation of the final densities along the bar. The SEM study showed that, with the system and conditions used, microstructures arising directly from spinodal decomposition could not be observed.

\section{ACKNOWLEDGMENTS}

A. Mühlebach, A. Hafner, and P.A. van der Schaaf (Ciba Specialty Chemicals Inc., Additives Research Dept., Basel, Switzerland) are gratefully acknowledged for synthesizing and kindly supplying the catalyst. The Swiss National Science Foundation Project No. 200053872.98 is gratefully acknowledged for financial support.

\section{REFERENCES}

1. A. Neubrand and J. Rödel, Z. Metallk. 88, 358 (1997).

2. A. Mortensen and S. Suresh, Int. Mater. Rev. 40, 239 (1995).

3. B.H. Rabin and I. Shiota, MRS Bull. 20, 32 (1995).

4. D. Prinz, V. Arnold, H-P. Buchkremer, A. Kuhstoss, P. Neumann, and D. Stöver, in Functionally Graded Materials 1998, conference held in New Town Hall, Dresden, Germany, Oct. 26-29, 1988, edited by W.A. Kaysser (Trans Tech Publications Ltd., Uetikon-Zuerich, Switzerland, 1999), p. 59.

5. W.A. Kaysser and B. Ilschner, MRS Bull. 20, 22 (1995).

6. R. Watanabe, MRS Bull. 20, 32 (1995).

7. C-W. Hong, in Functionally Graded Materials 1996, conference held in AIST Tsukuba Research Center, Tsukuba, Japan, Oct. 21-24, 1996, edited by I. Shiota and Y. Miyamoto (Elsevier Science B.V., Amsterdam, The Netherlands, 1997), p. 29.

8. I.P. Borovinskaya, A.G. Merzhanov, and V.I. Uvarov, in Functionally Graded Materials 1998, conference held in New Town Hall, Dresden, Germany, Oct. 26-29, 1998, edited by W.A. Kaysser (Trans Tech Publications Ltd., 1999), p. 151.

9. A. Neubrand, R. Jedamzik and J. Rödel, in Functionally Graded Materials 1996, conference held in AIST Tsukuba Research Center, Tsukuba, Japan, Oct. 21-24, 1996, edited by I. Shiota and Y. Miyamoto (Elsevier Science B.V., Amsterdam, The Netherlands, 1997), p. 233.

10. C.R. Choe, M. Park, and N-J. Lee, in Proc. Int. Conf. Comp. Mater. Energy (Technomic, Lancaster, Pa., 1995), p. 293.

11. M. Omori, H. Sakai, A. Okubo, and T. Hirai, in FGM '94 Symposium, edited by B. Ilschner and N. Cherradi, conference held in Lausanne, Switzerland, Oct. 1994 (Presses Polytechniques et Universitaires Romandes, Lausanne, Switzerland, 1994), p. 667.

12. M. Omori, A. Okubo, G.H. Kang, and T. Hirai, in Functionally Graded Materials 1996, edited by I. Shiota and Y. Miyamoto, conference held in AIST Tsukuba Research Center, Tsukuba, Japan, Oct. 21-24, 1996, (Elsevier Science B.V., Amsterdam, The Netherlands, 1997), p. 767.

13. T. Ishigure, E. Nihei, and Y. Koike, Appl. Opt. 33, 4261 (1994).

14. M. Kryszewski, Polym. Adv. Technol. 9, 244 (1998).

15. J-H. Liu and H-T. Liu, Polymer 38, 1251 (1997).

16. C.F. Jasso, J.J. Martinez, E. Mendizabal, and O. Laguna, J. Appl. Polym. Sci. 58, 2207 (1995).

17. G. Akovali, K. Biliyar, and M. Shen, J. Appl. Polym. Sci. 20, 2419 (1976).

18. K. Schulte and A. Poeppel, in Functionally Graded Materials 1998, edited by W.A. Kaysser, conference held in New Town Hall, Dresden, Germany, Oct. 26-29, 1998 (Trans Tech Publications Ltd., Uetikon-Zuerich, Switzerland, 1999), p. 101.
19. H. Ehbing and W. Michaeli, in Functionally Graded Materials 1998, edited by W.A. Kaysser, conference held in New Town Hall, Dresden, Germany, Oct. 26-29, 1998 (Trans Tech Publications Ltd., Uetikon-Zuerich, Switzerland, 1999), p. 107.

20. S. Pereira Nunes, Trends Polym. Sci. 5, 187 (1997).

21. A. Della Martina, J.G. Hilborn, and A. Mühlebach, Macromolecules 33, 2916 (2000).

22. K. Nakanishi, J. Porous Mater. 4, 67 (1997).

23. G.E. Elicabe, H.A. Larrondo and R.J.J. Williams, Macromolecules 31, 8173 (1998).

24. J. Borrajo, C.C. Riccardi, R.J.J. Williams, H.M. Siddiqi, M. Dumon, and J.P. Pascault, Polymer 39, 845 (1998).

25. E. Girard-Reydet, H. Sautereau, J.P. Pascault, P. Keates, P. Navard, G. Thollet, and G. Vigier, Polymer 39, 2269 (1998).

26. D. Chen, J.P. Pascault, H. Sautereau, and G. Vigier, Polym. Int. 32, 369 (1993).

27. P.A. Oyanguren, P.M. Frontini, R.J.J. Williams, G. Vigier, and J.P. Pascault, Polymer 37, 3087 (1996).

28. J. Kiefer, J.G. Hilborn, and J.L. Hedrick, Polymer 37, 5715 (1996).

29. J. Kiefer, J.G. Hilborn, J-A.E. Manson, Y. Leterrier, and J.L. Hedrick, Macromolecules 29, 4158 (1996).

30. J. Kiefer, J.G. Hilborn, J.L. Hedrick, H.J. Cha, D.Y. Yoon, and J.C. Hedrick, Macromolecules 29, 8546 (1996).

31. J. Kiefer, J.L. Hedrick and J.G. Hilborn, in Macromolecular Architectures, edited by J.G. Hilborn (Adv. Polym Sci. 147, Springer Verlag, Berlin, Germany, 1999), p. 161.

32. M.A. Bennett and A.K. Smith, J. Chem. Soc., Dalton Trans. 233 (1974).

33. ASTM standard D 1505-68.

34. W.J. Feast and D.B. Harrison, J. Mol. Catal. 65, 63 (1991).

35. B.M. Novak and R.H. Grubbs, J. Am. Chem. Soc. 110, 960 (1988).

36. B.M. Novak and R.H. Grubbs, J. Am. Chem. Soc. 110, 7542 (1988).

37. A. Mühlebach, P.A. Vanderschaaf, A. Hafner, and F. Setiabudi, J. Mol. Catal. A: Chem. 132, 181 (1998).

38. A. Hafner, A. Mühlebach, and P.A. Vanderschaaf, Angew. Chem., Int. Ed. Engl. 36, 2121 (1997).

39. A. Hafner, P.A. van der Schaaf, A. Mühlebach, P. Bernhard, U. Schaedeli, T. Karlen, and A. Ludi, Prog. Org. Coat. 32, 89 (1997).

40. D.A. Porter and K.E. Esterling, Phase Transformation in Metals and Alloys (Chapman \& Hall, London, U.K., 1992).

\section{APPENDIX A}

The gradient of 2-propanol concentration that should build up in the receiving flask can be predicted using the following approach.

In the system depicted by Fig. 1, let us consider that the concentration of the mixture poured in the flask at a certain time $t$ is the same as the one in bottom syringe at the same time $t$, neglecting the transit through the bottom needle. Let us also consider that the flows in and out of the bottom syringe, respectively named top and the bottom flows, are constant. In such a case, to obtain the concentration along the height of the bottle, it is sufficient to calculate the concentration variation with time in the bottom syringe and transform this result using the bottom flow and the dimensions of the flask. Considering Fig. 1 again, it can be observed that, in the bottom 
syringe, the only variation of volume of DCPD is due to the bottom flow. Thus the variation of volume of DCPD in the bottom syringe is given by

$$
\mathrm{d} V_{\mathrm{D}}=-C_{\mathrm{D}}(t) Q_{\mathrm{b}} \mathrm{d} t
$$

where $\mathrm{d} V_{\mathrm{D}}$ is the increment of volume of DCPD in the bottom syringe, $C_{\mathrm{D}}(t)$, the volumetric concentration of DCPD in the bottom syringe at time $t, Q_{\mathrm{b}}$, the bottom volume flow rate, and $\mathrm{d} t$, the increment of time.

In the present case

$$
C_{\mathrm{D}}(t)=\frac{V_{\mathrm{D}}(t)}{\mathrm{V}_{\text {tot }}(t)}
$$

where $V_{\mathrm{D}}(t)$ is the volume of DCPD in the bottom syringe at time $t$ and $V_{\text {tot }}(t)$ is the total volume in the bottom syringe at time $t$ and

$$
V_{\text {tot }}(t)=V_{0}+\left(Q_{\mathrm{t}}-Q_{\mathrm{b}}\right) t
$$

where $V_{\mathrm{I}}(t)$ is the volume of 2-propanol in the bottom syringe at time $t, V_{0}$, the initial volume in the bottom syringe, and $Q_{t}$, the top volume flow rate.

Replacing Eqs. (2) and (3) into Eq. (1) gives

$$
\frac{\mathrm{d} V_{\mathrm{D}}}{\mathrm{d} t}=-\frac{Q_{\mathrm{b}}}{V_{0}+\left(Q_{\mathrm{t}}-Q_{\mathrm{b}}\right) t} V_{\mathrm{D}}(t)
$$

Solving this first-order differential equation, with initial condition $V_{\mathrm{D}}(t=0)=V_{0}$, gives

$$
V_{\mathrm{D}}(t)=V_{0}\left(\frac{Q_{\mathrm{b}}}{Q_{\mathrm{t}}-Q_{\mathrm{b}}}+1\right)\left[V_{0}+\left(Q_{\mathrm{t}}-Q_{\mathrm{b}}\right) t\right]^{\left(-\frac{Q_{\mathrm{b}}}{Q_{\mathrm{t}}-Q_{\mathrm{b}}}\right)} .
$$

Introducing this result, together with Eq. (3), into Eq. (2) renders the volumetric concentration of DCPD in the bottom syringe:

$$
C_{\mathrm{D}}(t)=\left[\frac{V_{0}}{V_{0}+\left(Q_{\mathrm{t}}-Q_{\mathrm{b}}\right) t}\right]^{\left(\frac{Q_{\mathrm{b}}}{Q_{\mathrm{t}}-Q_{\mathrm{b}}}+1\right),},
$$

and obviously the volumetric concentration of 2-propanol in the bottom syringe, $C_{\mathrm{I}}(t)$, is

$$
C_{\mathrm{t}}(t)=1-\left[\frac{V_{0}}{V_{0}+\left(Q_{\mathrm{t}}-Q_{\mathrm{b}}\right) t}\right]\left(\frac{Q_{\mathrm{b}}}{Q_{\mathrm{t}}-Q_{\mathrm{b}}}+1\right) .
$$

Now the concentration of 2-propanol should be calculated versus the height in the sample. This can be done by correlating the level of mixture in the flask $h$ and time $t$ :

$$
\pi r_{\mathrm{f}}^{2} h=Q_{\mathrm{b}} t<=>t=\frac{\pi r_{\mathrm{f}}^{2} h}{Q_{\mathrm{b}}},
$$

where $r_{\mathrm{f}}$ is the radius of the base of the flask and $h$ is the height in the sample level of mixture.

Injecting this Eq. (8) into Eq. (7) gives

$$
C_{\mathrm{I}}(h)=1-\left[\frac{V_{0}}{V_{0}+\left(Q_{\mathrm{t}}-Q_{\mathrm{b}}\right) \frac{\pi r_{\mathrm{f}}^{2} h}{Q_{\mathrm{b}}}}\right]^{\left(\frac{Q_{\mathrm{b}}}{Q_{\mathrm{t}}-Q_{\mathrm{b}}}+1\right)} .
$$

